This is the post print version of the article, which has been published in VRST '18 Proceedings of the 24th ACM Symposium on Virtual Reality Software and Technology . 2018. Availabe online at

http://dx.doi.org/10.1145/3281505.3283375

\title{
Hands-free Vibrotactile Feedback for Object Selection Tasks in Virtual Reality
}

\author{
Tomi Nukarinen \\ TAUCHI, University of Tampere \\ tomi.nukarinen@uta.fi
}

\author{
Jari Kangas \\ TAUCHI, University of Tampere \\ jari.kangas@uta.fi
}

\author{
Jussi Rantala \\ TAUCHI, University of Tampere \\ jussi.e.rantala@uta.fi
}

\author{
Toni Pakkanen \\ TAUCHI, University of Tampere \\ toni.m.pakkanen@uta.fi
}

\author{
Roope Raisamo \\ TAUCHI, University of Tampere \\ roope.raisamo@uta.fi
}

\begin{abstract}
Interactions between humans and virtual environments rely on timely and consistent sensory feedback, including haptic feedback. However, many questions remain open concerning the spatial location of haptics on the user's body in VR. We studied how simple vibrotactile collision feedback on two less studied locations, the temples, and the wrist, affects an object picking task in a VR environment. We compared visual feedback to three visual-haptic conditions, providing haptic feedback on the participants' $(\mathrm{N}=16)$ wrists, temples or simultaneously on both locations. The results indicate that for continuous, hand-based object selection, the wrist is a more promising feedback location than the temples. Further, even a suboptimal feedback location may be better than no haptic collision feedback at all.
\end{abstract}

\section{CCS CONCEPTS}

- Human-centered computing $\rightarrow$ Virtual reality; Haptic devices; Pointing; Empirical studies in HCI;

\section{KEYWORDS}

Haptic feedback, visual feedback, collision detection, object selection in virtual reality

\section{ACM Reference Format:}

Tomi Nukarinen, Jari Kangas, Jussi Rantala, Toni Pakkanen, and Roope Raisamo. 2018. Hands-free Vibrotactile Feedback for Object Selection Tasks in Virtual Reality. In VRST 2018: 24th ACM Symposium on Virtual Reality Software and Technology (VRST '18), November 28-December 1, 2018, Tokyo, Japan. ACM, New York, NY, USA, 2 pages. https://doi.org/10.1145/3281505. 3283375

\section{INTRODUCTION}

As virtual reality is gaining popularity, it is increasingly relevant to investigate how to augment VR environments with haptics. We propose that it would be fruitful to study if simple haptic feedback could improve the user experience and efficiency of commercially

Permission to make digital or hard copies of part or all of this work for personal or classroom use is granted without fee provided that copies are not made or distributed for profit or commercial advantage and that copies bear this notice and the full citation on the first page. Copyrights for third-party components of this work must be honored.

For all other uses, contact the owner/author(s).

VRST '18, November 28-December 1, 2018, Tokyo, Japan

(C) 2018 Copyright held by the owner/author(s).

ACM ISBN 978-1-4503-6086-9/18/11.

https://doi.org/10.1145/3281505.3283375 available VR systems where virtual hand/3D cursor-based selection techniques are the norm. Haptic feedback has been found to increase performance compared to visual only feedback in many contexts of use [1]. Cheng et al. [2] showed that adding vibrotactile feedback to visual feedback improved task completion times in a grasping task. Moehring and Froehlich [3] demonstrated that adding tactile grasping feedback improved interaction in a CAVE and in using a head-mounted display. However, previous research [4] indicates that even in VR the usefulness of haptics is situational. We propose that the location of the feedback could be one such situational factor.

The motivation of this study was to explore vibrotactile feedback in VR for two less studied body locations that do not require a hand controller. The main contribution is in evaluating vibrotactile collision feedback in the wrist, and the temples for proximal, handbased object picking. Further, we demonstrate that in this specific context, the temples may be a suboptimal location for vibrotactile feedback.

\section{METHOD}

We explored the effect of vibrotactile collision detection feedback (i.e., feedback when the controller collides with an object) in an object picking act. There were four feedback conditions: visual only (No Haptics), haptic wrist (Wrist), haptic temples (Temples), and haptics on both the wrist and the temples (Both). We investigated if there are differences in user preferences or the speed of the interaction between the four feedback conditions.

16 volunteer participants ( 7 females, 9 males, mean age 37, SD 8.5, range $24-52$ years) from the university community took part in a user experiment. Ten out of 16 participants had at least some earlier experience of VR technology. One participant was left-handed, and 15 were right-handed. Five out of 16 participants wore eyeglasses during the experiment.

We used a laptop PC, an HTC Vive VR headset, an HTC Vive hand controller and Unity Virtual reality development environment to set up the experiment. As vibrotactile actuators, we used Minebea Linear Vibration Motors (LVM8, Matsushita Electric Industrial Co., Japan). These actuators were chosen mainly because their small size enables flexible use in different locations. We vibrated the actuators by sending audio signals from Unity to a Gigaport HD USB sound card, which connected to an IMG Stage Line STA-1508 amplifier. The haptic actuators were attached to a Velcro wristband and the HTC Vive headset (Figure 1). As Figure 1 shows, the actuators were located on the upper side of the wrist and near the left and right 

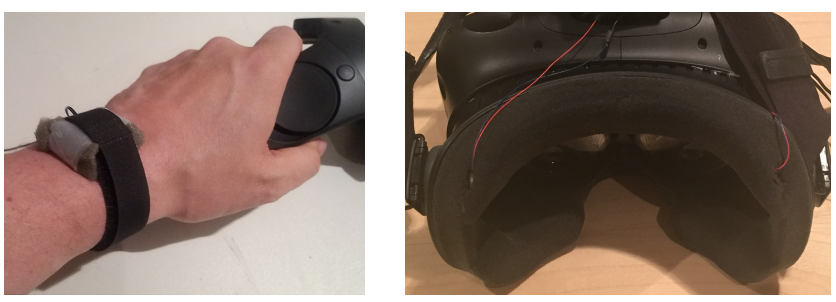

Figure 1: One actuator was on the wrist (left). Two actuators were on the participant's temples (right).
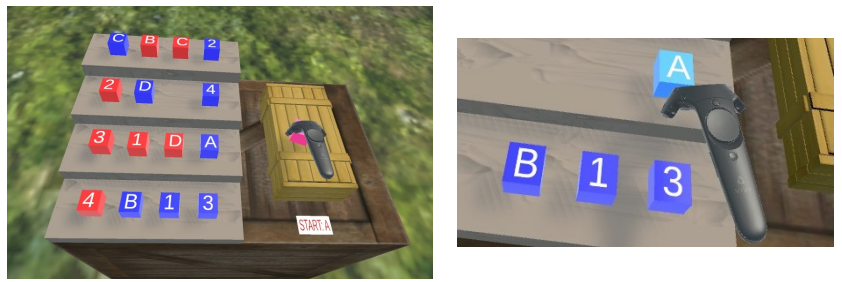

Figure 2: The grey picking tray and the brown dropping container (left). The visual collision detection feedback (right).

temples when worn by a user. As haptic feedback, we used $30 \mathrm{~ms}$ vibrotactile stimulation driven using a sine wave with a frequency of $150 \mathrm{~Hz}$. We utilized an object color changing visual feedback for the collision detection in all experimental conditions. (Figure 2, right).

A single trial consisted of moving sixteen randomized cubes from a tray to a separate container box (Figure 2, left) in a given order. The purpose was to move the controller over a cube, get feedback, press the controller trigger button to pick the cube, hold and move it over the container and drop the object by releasing the button. Each trial was repeated four times for each feedback condition. As we had four feedback conditions, the participants performed $256(16 \times 4 \times 4)$ object picks in the experiment. The participants answered a questionnaire about their experience after the vibrotactile conditions using a scale from -4 to 4 . Finally, we asked the participants to put the four conditions in preference order, and also explain why did they select the preferred method.

\section{RESULTS}

We analyzed six attributes of the vibrotactile feedback (control, arousal, pleasantness, effectiveness, strength, and timeliness) using Friedman tests, and did further pairwise comparisons with Wilcoxon tests. The Friedman tests showed statistically significant differences in the attributes control $(p=0.014)$, arousal $(p=0.043)$, pleasantness $(p=0.017)$, and effectiveness $(p=0.004)$. The tests did not show significant differences for the attributes strength $(p=$ $0.185)$ and timeliness $(p=0.819)$. Bonferroni-corrected Wilcoxon tests showed a significant difference on the feeling of control between the conditions Temples and Both $(p=0.045)$, and between Temples and Wrist $(p=0.036)$. The participants felt more control in the conditions Both and Wrist than in the condition Temples. There was also a statistically significant difference on the feeling of arousal $(p=0.027)$, pleasantness $(p=0.021)$, and effectiveness $(p=0.006)$ between the conditions Wrist and Temples. The participants rated the wrist feedback as more arousing, pleasant, and effective than the feedback on the temples.

For the most preferred method, the order was Wrist (62.5\%), Both (19\%), Temples (12.5\%), and No Haptics (6.25\%). For instance, $62.5 \%$ of the participants preferred the wrist condition the most. The order for least preferred method was No Haptics (62.5\%), Temples $(25 \%)$, and (Both) $(12.5 \%)$. We analyzed the differences on the rankings with a Friedman test and found a significant difference $\left(\chi^{2}(3)=30.23, p<0.001\right)$. Bonferroni-corrected Wilcoxon tests showed significant differences on the preference order between the conditions Wrist $(p=0.012)$ and No Haptics, and between Wrist and Temples $(p=0.024)$. The wrist feedback was significantly more preferred than either no haptics or haptics on the temples.

The most common comments about preferred feedback concerned the wrist haptics and how it felt most natural or realistic (5 comments), and how touching the object with a hand was felt on the hand (4). Also, wrist feedback was described as noticeable (2), supporting visual feedback (2), and making the task easier (2). Concerning combined feedback, two participants mentioned getting better confirmation. Further, two participants mentioned they could not feel the temple feedback. One participant out of 16 preferred visual feedback without haptics (condition No Haptics). The participant said that she felt more focused and faster without haptics. The comments on haptics were generally positive, and the participants said they would prefer to use the haptic feedback with VR devices.

For the trial completion time analysis, we did not identify outliers and therefore used the average from the four trials. The data were normally distributed, so we analyzed it with one-way repeated measures ANOVA. There were no statistically significant differences between the conditions $\left(F_{3,13}=0.19, p=0.903\right)$.

\section{CONCLUSION}

In sum, the wrist seems a more promising feedback location than the temples for continuous, hand-based object selection in a low cognitive load environment. We reason that temple actuation could be better for tasks with infrequent feedback, and when the tasks are closely tied to gaze and head movement behavior. Finally, the results suggest that a feedback location that is suboptimal for a task may still be a better choice than no haptic feedback at all.

\section{ACKNOWLEDGMENTS}

This work has received funding from the VARPU project (decision 1444/31/2016).

\section{REFERENCES}

[1] Jennifer L Burke, Matthew S Prewett, Ashley A Gray, Liuquin Yang, Frederick RB Stilson, Michael D Coovert, Linda R Elliot, and Elizabeth Redden. 2006. Comparing the effects of visual-auditory and visual-tactile feedback on user performance: a meta-analysis. In Proceedings of the 8th international conference on Multimodal interfaces. ACM, 108-117.

[2] Li-Te Cheng, Rick Kazman, and John Robinson. 1997. Vibrotactile feedback in delicate virtual reality operations. In Proceedings of the fourth ACM international conference on multimedia. ACM, 243-251.

[3] Mathias Moehring and Bernd Froehlich. 2011. Effective manipulation of virtual objects within arm's reach. In 2011 IEEE Virtual Reality Conference. 131-138. https://doi.org/10.1109/VR.2011.5759451

[4] Vijay M Pawar and Anthony Steed. 2009. Evaluating the influence of haptic force-feedback on 3D selection tasks using natural egocentric gestures. In Virtual Reality Conference, 2009. VR 2009. IEEE. IEEE, 11-18. 